\title{
Avaliação comparativa entre o modelo elétrico Battery e os modelos analíticos Linear e Lei de Peukert
}

\author{
Cleber Porciuncula ${ }^{1}$ \\ Alan Oliveira ${ }^{1}$ \\ Paulo S. Sausen ${ }^{1}$ \\ Airam Sausen ${ }^{1}$
}

\begin{abstract}
Resumo: O objetivo deste trabalho é realizar uma análise comparativa entre os resultados das simulações para o tempo de vida de uma bateria utilizada em dispositivos móveis, considerando os modelos analíticos Linear e Lei de Peukert e o modelo elétrico Battery. Os resultados das simulações são comparados com dados experimentais de uma bateria de íon lítio Nokia modelo BL-5F. A partir dos resultados das simulações, observou-se que a Lei de Peukert apresentou o melhor resultado, ou seja, um erro médio no tempo de vida de 1,96\%. Já o modelo elétrico Battery foi simulado considerando três curvas de calibração, sendo que, para a curva obtida a partir de uma corrente de descarga de $550 \mathrm{~mA}$, o seu melhor erro médio no tempo de vida foi de $2,60 \%$.
\end{abstract}

Palavras-chave: Baterias. Modelos matemáticos. Tempo de vida.

\begin{abstract}
The purpose of this paper is to perform a comparative analysis from simulation results for the battery lifetime used in mobile devices, considering the analytical models, Linear and Peukert's Law, and the Battery electric model. The results of the simulations are compared with experimental data of a Nokia BL-5F lithium-ion battery. From the results of the simulations it was observed that Peukert's Law showed the best result, i.e., an average error for the lifetime of $1.96 \%$, already the Battery electric model, was simulated considering three calibration curves, and for the curve obtained from a discharge current of $550 \mathrm{~mA}$ was obtained your best average error of lifetime that was of $2.60 \%$.
\end{abstract}

Keywords: Batteries. Lifetime. Mathematical models.

\section{Introdução}

As baterias eletroquímicas têm sido um dos principais focos de estudo e pesquisa para o ambiente de sistemas elétricos. Essas baterias, que alimentam a maioria dos dispositivos eletrônicos portáteis atuais, permitem armazenar energia em forma química para, posteriormente, convertê-la em energia elétrica, objetivando o fornecimento de energia a sistemas [1]. Atualmente, são inúmeras as áreas de aplicação dessas baterias, como, por exemplo, na área da saúde, em marca-passos; na área automotiva, em veículos híbridoelétricos; na área de dispositivos eletrônicos portáteis, em celulares, câmaras digitais, notebooks, ipads, entre outros.

Considerando o crescimento, nos últimos anos, na utilização de dispositivos eletrônicos portáteis, tem ocorrido um aumento na necessidade de baterias cada vez menores, mais leves e de melhor desempenho. Porém, essa evolução ainda não acompanha a progressiva demanda de energia e de limitação de tamanho, exigida pelos dispositivos móveis atuais [1].

\footnotetext{
${ }^{1}$ Mestrado em Modelagem Matemática, Unijuí, Campus Ijuí - Ijuí (RS) - Brasil.

\{cleber.porciuncula, alan.oliveira, sausen, airameunijui.edu.br\}
}

http://dx.doi.org/10.5335/rbca.2012.1989 
É importante destacar que o funcionamento de um dispositivo móvel eletrônico está condicionado ao estado de carga da bateria que o alimenta. Por esse motivo, no projeto deste dispositivo existe a preocupação com o tempo de vida da bateria, que é por definição o tempo que a mesma demora para atingir um determinado nível de capacidade de carga, denominado nível de cutoff. Quando esse nível é alcançado, a bateria não é mais capaz de fornecer energia elétrica ao sistema, sendo considerada descarregada. Surge, então, a necessidade de haver um método capaz de predizer o tempo de vida das baterias, e consequentemente, do sistema por elas alimentado. Uma das formas citadas na literatura de realizar essa predição é a partir da utilização de modelos matemáticos que simulam a descarga de energia das baterias.

Nesse contexto, o principal objetivo deste trabalho consiste em realizar um estudo comparativo entre o modelo elétrico Battery, presente no aplicativo MatLab/Simulink, e os modelos analíticos Linear e Lei de Peukert. Neste trabalho serão comparados os resultados obtidos a partir da simulação numérica com os resultados obtidos a partir de uma plataforma de testes [2], construída especialmente para avaliar modelos de descarga de baterias. A bateria utilizada para a execução dos experimentos reais e das simulações é a de íon lítio Nokia modelo BL-5F, utilizada na alimentação de telefones celulares da marca Nokia (e.g., Nokia N95).

O restante deste artigo está organizado da seguinte forma: na seção 2 é apresentada uma breve revisão do estado da arte dos modelos analíticos Linear e Lei de Peukert, bem como do modelo elétrico Battery; na seção 3 é apresentada a comparação entre os resultados das simulações dos modelos envolvidos com os resultados obtidos a partir da plataforma de testes; e, por fim, na seção 4 são apresentadas as conclusões do trabalho.

\section{Revisão dos modelos matemáticos}

Nesta seção são descritos e caracterizados dois modelos analíticos: Linear e Lei de Peukert e o modelo elétrico Battery. Observa-se que os modelos analíticos descrevem a bateria em um nível mais elevado de abstração, quando comparados com os modelos eletroquímicos, ou mesmo com os modelos elétricos [3].

Os modelos analíticos são considerados computacionalmente mais eficientes e flexíveis, requerendo avaliação de simples expressões analíticas, podendo ser facilmente estendidos para diferentes tipos de baterias. Destaca-se que nesses modelos as principais propriedades da bateria são modeladas utilizando-se um conjunto reduzido de equações, agregando acurácia e facilidade na sua implementação [4].

Dentre os modelos analíticos da literatura há os que são capazes de capturar os efeitos não lineares, tais como o efeito de recuperação e o efeito da taxa de capacidade, podendo incluir funções com cargas constantes e com cargas variáveis. Define-se por efeito de recuperação de uma bateria a reorganização dos elétrons no eletrólito no intervalo de tempo em que a corrente de descarga é nula, ou quando é reduzida significativamente, nesse caso uma maior quantidade de carga na superfície do eletrodo torna-se disponível, reequilibrando o sistema e a capacidade efetiva da bateria [3]. Já o efeito da taxa de capacidade depende da capacidade atual da bateria e da intensidade da corrente de descarga, ou seja, para correntes altas de descarga a capacidade efetiva da bateria é baixa, pois não há tempo suficiente para que ocorra o efeito de recuperação. Por outro lado, no caso de correntes de descargas alternadas, a capacidade efetiva da bateria é incrementada, pois na troca de uma corrente alta para uma corrente baixa, ou em períodos sem corrente, ocorre o efeito de recuperação [3].

Os modelos elétricos cuja precisão se situa entre a precisão dos modelos analíticos e dos modelos eletroquímicos (i.e., entre 1 e $5 \%$ de erro) são constituídos por uma combinação de fontes de tensão, resistores e capacitores para simularem as descargas de uma bateria. Para os profissionais da área de engenharia elétrica, estes modelos são mais intuitivos e fáceis de manipular, principalmente quando utilizados a partir de simuladores de circuito [1]. A seguir serão descritos os dois modelos analíticos e o modelo elétrico Battery utilizados.

\subsection{Modelo analítico Linear}

O modelo analítico mais simples é o modelo Linear $[3,5,6]$, cuja bateria é tratada como um recipiente linear de corrente. Tal modelo é descrito em (1), dado por

$$
C=C^{\prime}-I t_{d},
$$

onde $C$ é a capacidade restante da bateria, $C^{\prime}$ é a capacidade inicial, $I$ é a corrente constante de descarga e $t_{d}$ é o tempo de duração da operação. 
A capacidade remanescente é calculada sempre que a taxa de descarga for alterada. Dessa maneira, esse modelo não é capaz de capturar o efeito de recuperação.

\subsection{Lei de Peukert}

O modelo analítico mais simples, que considera parte das propriedades não lineares da bateria, é denominado Lei de Peukert [3, 5] descrita em (2) dada por

$$
L=\frac{a}{I^{b}}
$$

onde $L$ é o tempo de vida da bateria, $I$ é a corrente de descarga, e $a$ e $b$ são parâmetros que dependem do tipo de bateria utilizada, sendo obtidos a partir de dados experimentais através de alguma técnica de estimação de parâmetros. Neste trabalho é utilizada a técnica de Mínimos Quadrados.

Observa-se que essa lei captura a relação entre o tempo de vida e a taxa de descarga da bateria, por outro lado, não considera o efeito de recuperação. Em decorrência dessa característica os resultados da aplicação da Lei de Peukert, para a predição do tempo de vida da bateria, são razoáveis para cargas constantes, mas não para cargas variáveis [3].

\subsection{Modelo elétrico Battery}

Nesta seção é descrito o modelo elétrico Battery, disponibilizado no software Matlab, desenvolvido pela Mathworks, que proporciona um ambiente de trabalho interativo com linguagem própria de programação de alto nível, possibilitando ao usuário elaborar e solucionar tarefas complexas de forma mais rápida do que outras linguagens de programação, como C, C++ e Fortran.

O Matlab também disponibiliza uma série de caixas de ferramentas conhecidas como toolboxes ou blocksets. O Simulink é o mais conhecido gerador de diagramas de blocos do Matlab [7], gerando um ambiente de execução de simulações a partir da construção de modelos de projeto, baseado em sistemas dinâmicos ou embarcados. O ambiente de trabalho é gráfico e interativo, possibilitando ao usuário criar suas próprias bibliotecas de blocos para efetuar simulações, implementações e testes que demonstram o comportamento do sistema em relação à variação do tempo [8].

Uma biblioteca chamada SimPowerSystems, presente no aplicativo MatLab/Simulink, possui ferramentas para modelar e simular a geração, transmissão, distribuição e consumo de energia elétrica. Nesta biblioteca há um bloco chamado Battery, que é usado para implementar um modelo de bateria genérico, parametrizado, e dinâmico, a fim de representar os mais populares tipos de baterias recarregáveis (i.e., íon lítio, níquel cádmio, níquel metal hidreto e chumbo ácido). A partir do modelo elétrico Battery é possível simular o comportamento de carga ou descarga de um tipo específico de bateria, mediante a introdução de parâmetros no modelo [9].

Na Figura 1 é apresentado o diagrama de blocos simplificado do modelo elétrico Battery implementado no Matlab/Simulink. Observa-se que seu diagrama simplificado possui um resistor e uma fonte de tensão controlada. $\mathrm{O}$ resistor representa a resistência interna da bateria e a fonte de tensão controlada, a tensão elétrica nos seus terminais.

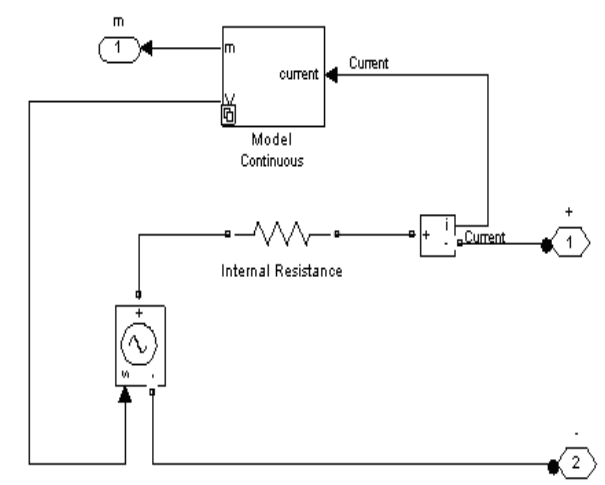

Figura 1: Ilustração do diagrama de blocos simplificado do modelo elétrico Battery [9] 
O bloco Model Continuous, presente no diagrama da Figura 1, utiliza os valores dos parâmetros da bateria para resolver equações matemáticas e a partir destas resoluções são simuladas as curvas de descarga da bateria.

A equação matemática do modelo elétrico Battery, que descreve o decaimento da tensão elétrica da bateria em um processo de descarga considerando uma bateria de íon lítio, é dada por (3)

$$
f\left(i t, i^{*}, i\right)=E_{0}-K \frac{Q}{Q-i t} i^{*}-K \frac{Q}{Q-i t} i t+A \exp (-B i t),
$$

onde $E_{0}$ é a tensão inicial considerando a bateria totalmente carregada, $K$ é a constante de polarização ou resistência de polarização, $i$ é a corrente dinâmica em baixa frequência, it é a capacidade extraída, $Q$ é a capacidade máxima da bateria, $A$ é a tensão exponencial e $B$ é a capacidade exponencial.

O modelo elétrico Battery possui uma série de hipóteses iniciais, dentre as quais se pode destacar as seguintes [9]:

- a resistência interna da bateria é considerada constante durante a descarga e não varia com a amplitude da corrente;

- a capacidade da bateria não muda com a amplitude da corrente de descarga;

- o modelo não leva em conta a temperatura;

- a autodescarga da bateria (i.e., perda de energia da bateria quando a mesma é armazenada por um longo tempo) não tem representação;

- o modelo não considera o efeito memória.

Para que o modelo elétrico Battery execute sua função de simulação de descarga de uma bateria e, consequentemente, de seu tempo de vida, os parâmetros apresentados na Tabela 1 devem ser informados [9].

Tabela 1: Parâmetros do modelo elétrico Battery

\begin{tabular}{ll}
\hline \multicolumn{1}{c}{ Parâmetros } & \multicolumn{1}{c}{ Descrição } \\
\hline Tipo de bateria & Informa a tecnologia da bateria. \\
Tensão nominal $(V)$ & Representa o final da zona linear das características de descarga. \\
Capacidade típica $(A h)$ & É a capacidade efetiva mínima da bateria. \\
SOC inicial $(\%)$ & Representa o estado inicial de carga da bateria. \\
Capacidade máxima $(A h)$ & É a capacidade teórica máxima $(Q)$, quando uma \\
descontinuidade ocorre na tensão da bateria.
\end{tabular}

\section{Comparação entre os modelos analíticos e o modelo elétrico Battery}

Nesta seção é apresentada uma comparação entre os resultados das simulações do tempo de vida de uma bateria de íon lítio Nokia modelo BL-5F de $950 \mathrm{mAh} / 3,7 \mathrm{~V}$, considerando os modelos analíticos Linear, Lei de Peukert e o modelo elétrico Battery, apresentados na seção 2 deste trabalho. Todos os resultados são comparados 
com dados experimentais obtidos a partir de uma plataforma de testes especialmente desenvolvida para esta finalidade.

A plataforma de testes é constituída por três componentes: sistema de controle, circuito e bateria. $\mathrm{O}$ sistema de controle (software) apresenta uma interface intuitiva, disponibilizando a partir de gráficos as informações coletadas pelos sensores de tensão e corrente. O circuito é composto por um microcontrolador e possui a tarefa de realizar a comunicação com o computador, bem como de administrar os módulos de sensoriamento e controle de descarga [2]. Na Figura 2 é apresentada uma foto da plataforma de testes utilizada neste trabalho. Mais detalhes sobre o funcionamento da plataforma podem ser obtidos em Nonemacher [2].

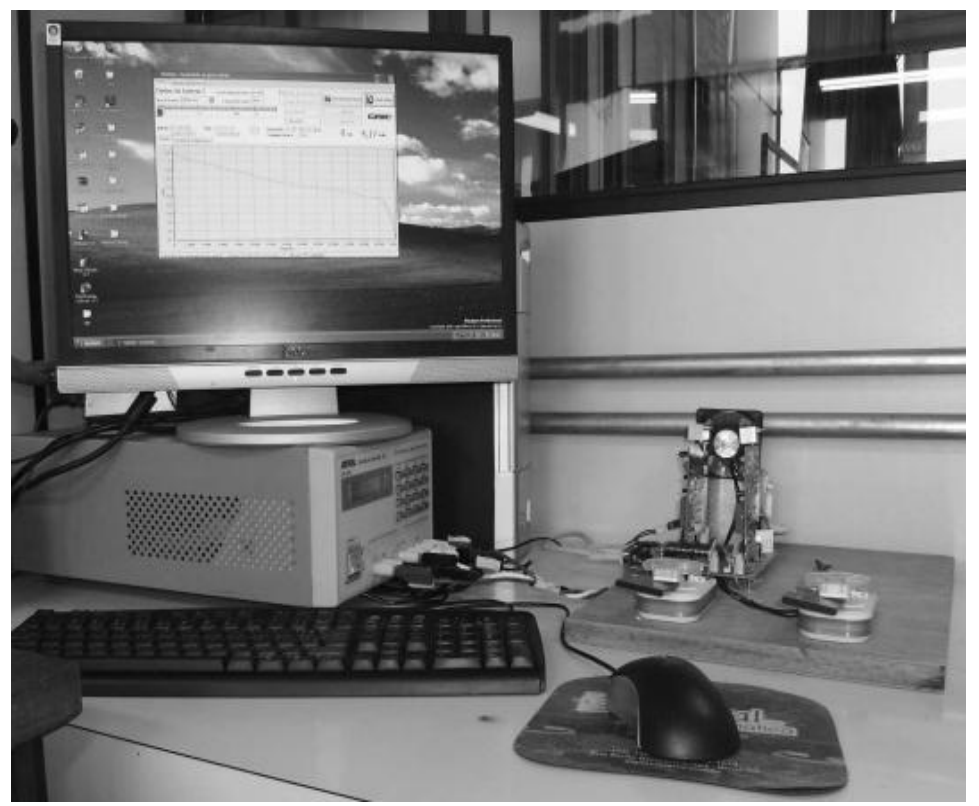

Figura 2: Foto da plataforma de testes

Nos testes experimentais foi realizada uma série de ensaios utilizando uma bateria de íon lítio Nokia modelo BL-5F, empregando-se uma metodologia na qual foram utilizadas dez descargas contínuas, e para cada uma dessas descargas foram realizados dez ensaios, objetivando a obtenção de uma amostragem estatística satisfatória. A partir desses ensaios foi possível calcular as médias do tempo de vida para cada descarga contínua [10]. Os tempos médios de vida, expressos em minutos $(\mathrm{min})$, para cada descarga estão apresentados na Tabela 2.

Na Figura 3 são apresentadas as dinâmicas de tensão elétrica na bateria, considerando os processos de descargas contínuas, a partir de dez curvas que foram obtidas a partir dos tempos médios de vida para cada um dos perfis de descarga apresentados na Tabela 2. Analisando o gráfico, observou-se que o tempo de vida da bateria diminui à medida que a corrente de descarga aumenta. Este fato ocorre porque para altas taxas de descarga a capacidade efetiva da bateria é reduzida, e, consequentemente, seu tempo de vida também. Isso decorre em virtude de não haver tempo suficiente para que ocorra o efeito de recuperação na bateria.

Tabela 2: Tempos médios de vida (Tmv) para cada descarga contínua da bateria Nokia BL-5F [10]

\begin{tabular}{ccccccccccc}
\hline $\begin{array}{c}\text { Perfis } \\
(m A)\end{array}$ & 100 & 150 & 250 & 350 & 450 & 550 & 650 & 750 & 850 & 950 \\
\hline $\begin{array}{c}\text { Tmv } \\
(\text { min. })\end{array}$ & 483,71 & 325,67 & 192,32 & 136,25 & 105,70 & 85,80 & 70,73 & 60,74 & 51,70 & 46,70 \\
\hline
\end{tabular}




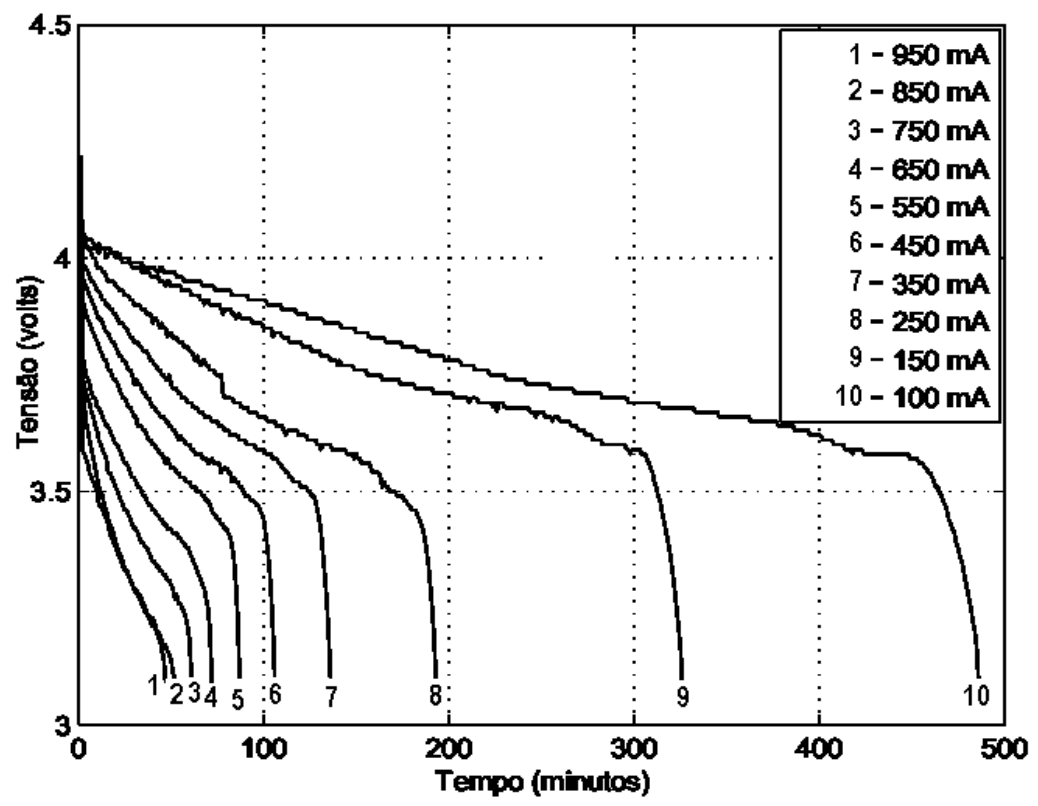

Figura 3: Curvas de tensão em função do tempo de descarga [10]

Conforme descrito na seção 2, em (2) está descrita a Lei de Peukert para descargas contínuas, apresentando dois parâmetros $a$ e $b$ que dependem do tipo de bateria utilizado e podem ser estimados através da técnica de mínimos quadrados, considerando os experimentos realizados na plataforma de testes. Nesse contexto, após a realização deste procedimento, foram encontrados os seguintes valores para os parâmetros $a=53183$ e $b=1,0195[10]$.

Em seguida, foram realizadas as simulações do modelo Linear e da Lei de Peukert, considerando os dez perfis de descargas e os dados obtidos a partir da plataforma de testes. Na Tabela 3 são apresentados os tempos de vida, em minutos (min), encontrados pela aplicação dos dois modelos analíticos em conjunto com seus erros médios em relação aos dados experimentais apresentados na Tabela 2.

Tabela 3: Resultados simulados e erros dos modelos analíticos [10]

\begin{tabular}{ccccc}
\hline \multirow{2}{*}{ Descarga contínua $(m A)$} & \multicolumn{2}{c}{ Modelo Linear } & \multicolumn{2}{c}{ Lei de Peukert } \\
& Tempo (min) & Erro (\%) & Tempo (min) & Erro (\%) \\
\hline 100 & 570,02 & 17,84 & 486,15 & 0,51 \\
150 & 380 & 16,68 & 321,55 & 1,26 \\
250 & 228 & 18,55 & 191,02 & 0,68 \\
350 & 162,87 & 19,54 & 135,55 & 0,51 \\
450 & 126,67 & 19,84 & 104,91 & 0,74 \\
550 & 103,65 & 20,8 & 85,5 & 0,35 \\
650 & 87,7 & 24 & 72,11 & 1,96 \\
750 & 76 & 25,13 & 62,32 & 2,61 \\
850 & 67,07 & 29,74 & 54,86 & 6,11 \\
950 & 60 & 28,49 & 48,98 & 4,88 \\
\hline Erro médio (\%) & \multicolumn{4}{|c|}{} \\
\hline
\end{tabular}

Para a realização das simulações do tempo de vida a partir do modelo elétrico Battery, os parâmetros do modelo podem ser obtidos a partir de uma única curva de descarga da bateria de íon lítio Nokia BL-5F denominada curva de calibração, em conjunto com os dados do seu datasheet. Neste trabalho, objetivando verificar a partir de qual curva o modelo elétrico Battery apresenta os melhores resultados de simulação, foram escolhidas três curvas de calibração a partir das descargas contínuas de $150 \mathrm{~mA}, 550 \mathrm{~mA}$ e $950 \mathrm{~mA}$. Os parâmetros obtidos a partir dessas três curvas, bem como os dados de seu datasheet, são apresentados na Tabela 4.

Tabela 4: Parâmetros utilizados nas simulações de descarga da bateria de íon lítio BL-5F 


\begin{tabular}{lccc}
\hline \multicolumn{1}{c}{ Parâmetros } & $\begin{array}{c}\text { Descarga de } \\
\mathbf{1 5 0 ~} \mathrm{mA}\end{array}$ & $\begin{array}{c}\text { Descarga de } \\
\mathbf{5 5 0} \mathrm{mA}\end{array}$ & $\begin{array}{c}\text { Descarga de } \\
\mathbf{9 5 0} \mathrm{mA}\end{array}$ \\
\hline Tensão nominal ou cutoff & $3,10 \mathrm{~V}$ & $3,10 \mathrm{~V}$ & $3,10 \mathrm{~V}$ \\
Capacidade típica & $0,950 \mathrm{Ah}$ & $0,950 \mathrm{Ah}$ & $0,950 \mathrm{Ah}$ \\
Estado de carga & $100 \%$ & $100 \%$ & $100 \%$ \\
Capacidade maxima & $0,9975 \mathrm{Ah}$ & $0,9975 \mathrm{Ah}$ & $0,9975 \mathrm{Ah}$ \\
Tensão com carga completa & $4,17 \mathrm{~V}$ & $4,17 \mathrm{~V}$ & $4,17 \mathrm{~V}$ \\
Corrente de descarga nominal & $0,150 \mathrm{~A}$ & $0,550 \mathrm{~A}$ & $0,950 \mathrm{~A}$ \\
Resistência interna & $0,02945 \mathrm{Ohms}$ & $0,02945 \mathrm{Ohms}$ & $0,02945 \mathrm{Ohms}$ \\
Capacidade na tensão nominal & $0,8129 \mathrm{Ah}$ & $0,7912 \mathrm{Ah}$ & $0,7365 \mathrm{Ah}$ \\
Tensão exponencial & $3,7 \mathrm{~V}$ & $3,7 \mathrm{~V}$ & $3,7 \mathrm{~V}$ \\
Capacidade exponencial & $0,5032 \mathrm{Ah}$ & $0,2105 \mathrm{Ah}$ & $0,0240 \mathrm{Ah}$ \\
Unidade & $s$ & $\mathrm{~s}$ & $\mathrm{~s}$ \\
Tempo de resposta & $30 \mathrm{~s}$ & $30 \mathrm{~s}$ & $30 \mathrm{~s}$ \\
\hline
\end{tabular}

Para as simulações do modelo elétrico Battery, na ferramenta computacional Matlab/Simulink, é utilizado o método numérico de Bogacki Shampine com o tamanho do passo fixado em 1 segundo. Nas simulações, a resistência interna da bateria é considerada constante. Em razão disso, para a definição do parâmetro de resistência interna, foi utilizado um valor que corresponde a $1 \%$ do produto da tensão nominal ou cutoff pela capacidade típica da bateria, conforme proposto no site da MathWorks [9]. O valor do parâmetro capacidade máxima foi considerado como $105 \%$ da capacidade típica da bateria e o tempo de resposta como 30s, conforme também proposto no site da MathWorks [9].

No primeiro momento, as simulações do modelo elétrico Battery foram realizadas considerando uma corrente nominal de descarga de $150 \mathrm{~mA}$ e parâmetros extraídos da curva experimental referente a uma descarga contínua de $150 \mathrm{~mA}$. Logo após, foram realizadas simulações considerando uma corrente nominal de descarga de $550 \mathrm{~mA}$ e parâmetros extraídos da curva experimental referente à descarga contínua de $550 \mathrm{~mA}$. E, por fim, foram realizadas simulações considerando uma corrente nominal de descarga de $950 \mathrm{~mA}$ e parâmetros extraídos da curva experimental obtida a partir de uma descarga contínua de $950 \mathrm{~mA}$.

Nas Tabelas 5, 6 e 7 são apresentados os tempos de vida médios da bateria de íon lítio Nokia modelo BL$5 \mathrm{~F}$ obtidos de forma experimental e os tempos de vida estimados, obtidos a partir de simulações realizadas com o modelo elétrico Battery, considerando as correntes nominais de descarga de $150 \mathrm{~mA}, 550 \mathrm{~mA}$ e $950 \mathrm{~mA}$, respectivamente.

A aproximação entre os tempos de vida experimentais e simulados nas Tabelas 5, 6 e 7 indica que o modelo elétrico Battery prediz o tempo de vida da bateria com uma precisão razoável. Observa-se que para as três calibrações consideradas (i.e., com as correntes nominais de descarga de $150 \mathrm{~mA}, 550 \mathrm{~mA}$ e $950 \mathrm{~mA}$ ) o modelo continua apresentando um erro médio que não ultrapassa $5 \%$, erro esperado no caso de modelos elétricos [1].

Tabela 5: Resultados experimentais e simulados (150 mA)

\begin{tabular}{cccc}
\hline \multirow{2}{*}{ Descarga contínua $(m A)$} & Experimento & \multicolumn{2}{c}{ Simulação } \\
& Tempo de vida $(s)$ & Tempo de vida $(s)$ & Erro no tempo de vida $(\%)$ \\
\hline 100 & 29022,6 & 29565 & 1,88 \\
250 & 11539,2 & 11467 & 0,63 \\
350 & 8175 & 8019 & 1,91 \\
450 & 6342 & 6103 & 3,77 \\
550 & 5148 & 4882 & 5,17 \\
650 & 4243,8 & 4037 & 4,87 \\
750 & 3644,4 & 3417 & 6,24 \\
850 & 3102 & 2942 & 5,16 \\
\hline
\end{tabular}




\begin{tabular}{cccc}
\hline 950 & 2802 & 2567 & 8,39 \\
\hline \multicolumn{4}{c}{ Erro médio (\%) } \\
\hline \\
Tabela 6: Resultados experimentais e simulados $(550 m A)$ \\
\hline Descarga contínua $(m A)$ & Experimento & Simulação \\
\hline 100 & Tempo de vida $(s)$ & Tempo de vida $(s)$ & Erro no tempo de vida $(\%)$ \\
150 & 29022,6 & 30732 & 5,89 \\
250 & 19540,2 & 20324 & 4,01 \\
350 & 11539,2 & 11997 & 3,97 \\
450 & 8175 & 8428 & 3,09 \\
650 & 6342 & 6444 & 1,61 \\
750 & 4243,8 & 4305 & 1,44 \\
850 & 3644,4 & 3663 & 0,51 \\
950 & 3102 & 3171 & 2,22 \\
& 2802 & 2783 & 0,68 \\
\hline
\end{tabular}

Tabela 7: Resultados experimentais e simulados $(950 \mathrm{~mA})$

\begin{tabular}{cccc}
\hline \multirow{2}{*}{ Descarga contínua $(m A)$} & $\begin{array}{c}\text { Experimento } \\
\text { Tempo de vida }(s)\end{array}$ & Tempo de vida $(s)$ & $\begin{array}{c}\text { Simulação } \\
\text { Erro no tempo de vida }(\%)\end{array}$ \\
\hline 100 & 29022,6 & 30779 & 6,05 \\
150 & 19540,2 & 20357 & 4,18 \\
250 & 11539,2 & 12019 & 4,16 \\
350 & 8175 & 8445 & 3,30 \\
450 & 6342 & 6458 & 1,83 \\
550 & 5148 & 5193 & 0,87 \\
650 & 4243,8 & 4317 & 1,72 \\
750 & 3644,4 & 3674 & 0,81 \\
850 & 3102 & 3181 & 2,55 \\
\hline
\end{tabular}

Considerando que a bateria de íon lítio utilizada possui uma capacidade típica de $950 \mathrm{mAh}$, para as simulações primeiramente foi feita a calibração do modelo com parâmetros baseados em uma corrente nominal de descarga baixa (150 $\mathrm{mA})$, posteriormente em uma corrente nominal intermediária $(550 \mathrm{~mA})$ e finalmente em uma corrente nominal alta $(950 \mathrm{~mA})$. O modelo apresentou um erro médio menor quando sua calibração foi realizada com parâmetros da curva experimental de descarga baseada na corrente nominal de descarga de $550 m A$. Este erro foi de $2,6 \%$.

Observa-se também, principalmente nas Tabelas 5 e 6, que os erros no tempo de vida apresentados pelo modelo aumentam na medida em que os valores das descargas contínuas se afastam do valor da corrente nominal de descarga utilizada para calibrar o modelo. Embora isso não esteja evidente na Tabela 6, nota-se que os erros apresentados em cada uma das três tabelas possuem uma diferença considerável entre o mínimo e o máximo de erro no tempo de vida. Isso acontece justamente em razão do modelo elétrico Battery não capturar um efeito não linear importante que ocorre na bateria durante seu processo de descarga, chamado de "efeito de taxa de capacidade". Apesar disso, o erro médio no tempo de vida do modelo elétrico Battery ficou bastante próximo do erro médio da Lei de Peukert, quando as simulações foram realizadas com parâmetros extraídos da curva de descarga de $550 \mathrm{~mA}$, e consequentemente, com a corrente nominal de descarga de $550 \mathrm{~mA}$.

É importante destacar que todas as simulações executadas pelo modelo elétrico Battery podem ser baseadas em uma única curva experimental de descarga. Os parâmetros obtidos a partir dessa curva são utilizados para inicializar o modelo elétrico, e após essa inicialização basta alterar a corrente de descarga a ser aplicada no modelo para que simule outro tempo de vida. No entanto, neste trabalho, as simulações foram baseadas em três curvas de descarga, objetivando verificar a partir de qual delas o modelo apresentou melhores resultados simulados. 
A Lei de Peukert apresentou bons resultados. Esse fato se deve por este modelo analítico considerar uma parte dos efeitos não lineares que ocorrem durante o período de descarga da bateria, bem como por terem sido realizadas uma quantidade significativa de testes experimentais de descarga (i.e., dez neste caso), utilizadas para a estimação de seus parâmetros. Caso fosse utilizado um número reduzido de curvas experimentais para esta estimação, maiores seriam os erros na estimação do tempo de vida da bateria.

O modelo Linear apresentou os piores resultados, quando comparados com a Lei de Peukert. Mesmo que as simulações tenham sido baseadas em dados experimentais, o modelo Linear não apresenta bons resultados em decorrência de não considerar os efeitos não lineares da bateria, que ocorrem nas operações físicas de descarga, e que influenciam diretamente em sua capacidade, e consequentemente na estimação do seu tempo de vida.

\section{Conclusão}

Neste trabalho foi realizada a análise e a comparação do modelo elétrico Battery, presente na ferramenta computacional MatLab/Simulink, com dois modelos analíticos encontrados na literatura, o modelo Linear e a Lei de Peukert. Os resultados das simulações foram avaliados a partir da comparação com dados reais obtidos de uma plataforma de testes.

Foram apresentados os resultados das simulações do tempo de vida dos modelos analíticos e, logo em seguida, os resultados simulados do modelo elétrico Battery, considerando três correntes nominais de descarga (i.e., $150 \mathrm{~mA}, 550 \mathrm{~mA}$ e $950 \mathrm{~mA}$ ). O menor erro médio na estimação do tempo de vida apresentado pelo modelo elétrico Battery foi de 2,60\% quando utilizados os parâmetros obtidos a partir da corrente nominal de descarga de $550 \mathrm{~mA}$. Este erro médio está dentro dos limites esperados para um modelo elétrico e está próximo do erro médio da Lei de Peukert, que foi de $1,96 \%$.

Em contrapartida, conforme Schneider [10], foram necessárias dez curvas experimentais de descarga para a estimação dos parâmetros da Lei de Peukert, o que evidencia uma maior dificuldade na obtenção dos parâmetros para este modelo. Nesse contexto, pode-se concluir que o modelo elétrico Battery apresenta bons resultados na estimação do tempo de vida da bateria considerada, utilizando-se somente três curvas experimentais de descarga para a obtenção de seus parâmetros.

Como trabalhos futuros, pretende-se estender este trabalho, adicionando a simulação de descargas variáveis objetivando avaliar o comportamento do modelo elétrico Battery e dos modelos analíticos Linear e Lei de Peukert para este cenário.

\section{Referências}

[1] CHEN, M.; RINCÓN-MORA G. A. Accurate electrical Battery model capable of predicting runtime and I V Performance. IEEE Transactions on Energy Conversion, vol. 21, no. 2, p. 504-511, 2006.

[2] NONEMACHER, H. B. et al. Desenvolvimento de um Testbed para avaliação de modelos matemáticos utilizados na predição do tempo de vida de baterias. In: UNIVERSIDADE FEDERAL DE RIO GRANDE, 24, 2010, Rio Grande, Brasil. Anais do Congresso Regional de Iniciação Científica e Tecnológica em Engenharia. Rio Grande, 2010. p. 2-4.

[3] JONGERDEN, M. R.; HAVERKORT, B. R. H. M. Which battery model to use? In: UK PERFORMANCE ENGINEERING WORKSHOP, 24th, 2008, London, UK. Technical Report Series of the Department of Computing, Imperial College London (DTR08-9). Imperial College London, 2008. p. 76-88.

[4] LAHIRI, K. et al. Battery-driven system design: a new frontier in low power design. In: ASIA AND SOUTH PACIFIC AND THE INTERNATIONAL CONFERENCE ON VLSI DESIGN, 7th, 2002. Proceedings of ASP-DAC, 2002. p. 261-267.

[5] JONGERDEN, M. R.; HAVERKORT, B. R. H. M. Battery modeling. Technical Report in Faculty Electrical Engineering, Mathematics and Computer Science (EEMCS), 2008. 
[6] SAUSEN, P. S. Gerenciamento integrado de energia e controle de topologia em redes de sensores sem fio. 2008. Tese (Doutorado em Engenharia Elétrica) - Universidade Federal de Campina Grande - Campina Grande, Paraíba, Brasil.

[7] Mathworks on-line. Disponível em: <http://www.mathworks.com/products/matlab>. Acesso em: 29 nov. 2010.

[8] Mathworks online. Disponível em: < http://www.mathworks.com/products/simulink>. Acesso em: 29 nov. 2010.

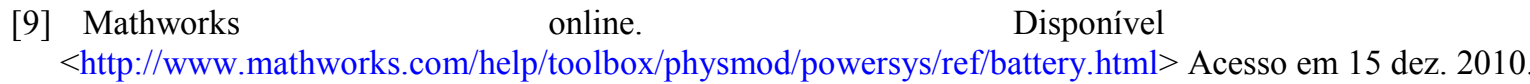

[10] SCHNEIDER, K. K. Avaliação de modelos analíticos na predição do tempo de vida de baterias utilizadas em dispositivos móveis, 2011. Dissertação (Mestrado em Modelagem Matemática) - Universidade Regional do Noroeste do Estado do Rio Grande do Sul, Ijuí, RS, Brasil. 